\title{
Neural circuitry of emotion regulation: Effects of appraisal, attention, and cortisol administration
}

\author{
Sean T. Ma ${ }^{1}$ - James L. Abelson ${ }^{1} \cdot$ Go Okada $^{2} \cdot$ Stephan F. Taylor $^{1}$ • Israel Liberzon ${ }^{1,3,4}$
}

Published online: 28 December 2016

(C) Psychonomic Society, Inc. 2016

\begin{abstract}
Psychosocial well-being requires effective regulation of emotional responding in context of threat or stress. Neuroimaging studies have focused on instructed, volitional regulation (e.g., reappraisal or distancing), largely ignoring implicit regulation that does not involve purposeful effort to alter emotional experience. These implicit processes may or may not involve the same neural pathways as explicit regulatory strategies. We examined the neurobiology of implicit emotional regulation processes and the impact of the stress hormone cortisol on these processes. Our study task employed composite pictures of faces and places to examine neural activity during implicit emotional processing (of emotional faces), while these responses were implicitly regulated by attention shift away from the emotionally evocative stimuli, and while subjects reflectively appraised their own emotional response to them. Subjects completed the task in an fMRI scanner after random assignment to receive placebo or hydrocortisone (HCT), an orally administered version of cortisol. Implicit emotional processing activated insula/IFG, $\mathrm{dACC} / \mathrm{dMPFC}$, midbrain and amygdala. With attention
\end{abstract}

Electronic supplementary material The online version of this article (doi:10.3758/s13415-016-0489-1) contains supplementary material, which is available to authorized users.

Israel Liberzon

liberzon@umich.edu

1 Department of Psychiatry, University of Michigan, Ann Arbor, MI 48109-2701, USA

2 Department of Psychiatry, Hiroshima University, Hiroshima, Japan

3 Mental Health Service, Veterans Affairs Ann Arbor Health System, Ann Arbor, MI 48105, USA

4 Department of Psychiatry, University of Michigan, 4250 Plymouth Road, Ann Arbor, MI 48109-2700, USA shifting, we saw diminished signal in emotion generating/ response regions (e.g., amygdala) and increased activations in task specific attention regions like parahippocampus. With appraisal of emotions, we observed robust activations in medial prefrontal areas, where activation is also seen in instructed reappraisal studies. We observed no main effects of HCT administration on brain, but males and females showed opposing neural effects in prefrontal areas. The data suggest that different types of emotion regulation utilize overlapping circuits, but with some strategy specific activation. Further study of the dimorphic sex response to cortisol is needed.

Keywords Emotion $\cdot$ Regulation $\cdot$ Attention $\cdot$ Cognitive control $\cdot$ Prefrontal cortex

Effective emotion regulation is crucial for psychosocial wellbeing (Berking \& Wupperman, 2012; Eisenberg, 2000; Gross \& Munoz, 1995). A growing body of neuroscientific work examines the psychological and neurobiological mechanisms that contribute to effective regulation of emotions (Gross, 1998; Ochsner \& Gross, 2007). The bulk of this work studies explicit and volitional regulation processes, such as distancing and reappraisal (McRae et al., 2010). In these studies, participants are asked to purposely alter responses to emotion-eliciting stimuli by cognitively separating themselves from the perceived cues (distancing) or by reinterpreting what they are seeing (reappraisal), e.g., by reframing a gory scene as coming from a movie rather than reflecting real injury. These techniques reduce emotional reactivity, increase neural activity in regulatory brain regions such as medial prefrontal cortex ( $\mathrm{mPFC}$ ), dorsolateral PFC (dlPFC), and anterior cingulate cortex (ACC), and reduce activity in salience detection regions such as amygdala (Kompus, Hugdahl, Ohman, Marklund, \& Nyberg, 2009; Ochsner \& Gross, 2007). They are of considerable clinical 
interest because they parallel techniques used in cognitivebehavioral therapies (Hannesdottir \& Ollendick, 2007; Porto et al., 2009) and because these same brain regions may play a pathophysiological role in mood/anxiety disorders (Shang et al., 2014). However, instructed regulatory efforts do not capture all available "real life" regulation processes, some of which are more implicit and do not involve volitional effort or techniques specifically designed to modulate emotion (Kuhn, Haggard, \& Brass, 2014).

There are in fact other processes that can impact emotional responses without requiring explicit effort to alter emotional experience or expression. Attention allocation and appraisal of emotion are two examples of ubiquitous, ecologically valid processes that can impact emotional processing, and may be potential points of intervention for enhancing emotional regulation capacities (Lieberman et al., 2007; S. F. Taylor, Phan, Decker, \& Liberzon, 2003). Attentional control may be the simplest example, with evidence that shifting attention away from emotionally arousing components of a stimulus can reduce emotional reactivity to it, with potential therapeutic value (Almeida et al., 2014; Britton et al., 2013). In the same vein, when emotionally arousing stimuli trigger emotional responses, a simple reflection or cognitive assessment of this response can diminish its intensity even if no volitional "regulation" was intended. The intensity of feelings elicited can be reduced by simply asking subjects to appraise their feelings (a form of internally directed cognitive appraisal) and choose a label for them (Liberzon et al., 2000; Lieberman et al., 2007; Payer, Baicy, Lieberman, \& London, 2012; S. F. Taylor et al., 2003).

Whether these processes should be defined as emotion regulation can be debated, but additional data on the neural processes involved can help elucidate the nature of emotion and the boundaries between emotion generation and emotion regulation processes (Gross \& Barrett, 2011). If attention shifting and this type of appraisal can alter emotions and they do so via the same neural pathways involved in more traditional regulation strategies, then they can perhaps be thought of as implicit emotion regulation strategies because they do not involve instructed, volitional efforts to directly alter emotional responses. Available evidence suggests that they may in fact impact emotional processing through similar pathways as more explicit, volitional regulation strategies. Labeling, for example, increases mPFC activity (Phan, Wager, Taylor, \& Liberzon, 2002; S. F. Taylor et al., 2003), and simultaneously reduces amygdala activation (Critchley et al., 2000; Hariri, Bookheimer, \& Mazziotta, 2000; Hariri, Mattay, Tessitore, Fera, \& Weinberger, 2003; Liberzon et al., 2000; Lieberman et al., 2007; S. F. Taylor et al., 1998; S. F. Taylor et al., 2003), which parallels findings on explicit emotion regulation strategies (McRae et al., 2010). Attention shifting has not been specifically studied as an emotion regulation strategy, but attentional control involves activity in dIPFC and ACC, which modulate activity in salience processing regions like amygdala (Bishop, 2008; Klumpp, Angstadt, \& Phan, 2012), again paralleling pathways involved in explicit emotion regulation. Clinically useful attention training can also increase PFC activation and reduce activation in salience processing regions like amygdala (C. T. Taylor et al., 2014).

Attention and appraisal processes have relevance to psychiatric disorders like PTSD and social anxiety disorder, where they may be involved in pathophysiology (Britton, Lissek, Grillon, Norcross, \& Pine, 2011; Elsesser, Freyth, Lohrmann, \& Sartory, 2009) and might provide additional targets for intervention development (Britton et al., 2013; Britton et al., 2014; Browning, Holmes, Murphy, Goodwin, \& Harmer, 2010). Identifying the pathways through which they work will help us understand their potential roles in both pathophysiology and treatment and help determine whether they should be thought of as emotion regulation approaches with therapeutic potential. If they alter emotional experience, via the same neural circuits as explicit, volitional emotion regulation techniques (Aron, Robbins, \& Poldrack, 2004; Quirk \& Gehlert, 2003) - they should perhaps be thought of as emotion regulation processes and further studied as such. To further explore these processes, we adapted an attention shifting task (Anderson, Christoff, Panitz, De Rosa, \& Gabrieli, 2003) to incorporate two different implicit emotion regulation mechanisms, attention-shifting and appraisal of emotions (using a labeling-like task), further modified to allow us to examine the effects of these processes on known emotion regulation pathways in the brain.

Emotion generation and emotion regulation are critically involved in adaptive behavioral responding to environmental threat and challenge. They are often called upon in situations of acute stress, with important implications for organismic outcomes. They influence and are influenced by activity in the body's central, neuroendocrine stress response system, the hypothalamic-pituitary adrenal (HPA) axis. The end product of HPA axis activation, cortisol, acutely influences a host of neurocognitive processes relevant to fear, learning, and coping (Buchanan \& Lovallo, 2001; De Quervain, Aerni, Schelling, \& Roozendaal, 2009; Payne et al., 2007). These effects are likely mediated by neural circuits that include mPFC and amygdala (as well as hippocampus), where the glucocorticoid receptors are densely distributed (Sarrieau et al., 1986; Watzka et al., 2000). Cortisol modulation of functional connectivity between amygdala and mPFC (Henckens, van Wingen, Joels, \& Fernandez, 2010; Veer et al., 2012), for example, offers a potential pathway for its effects on emotion regulation. Furthermore, dysregulation of HPA axis activity is well documented in numerous psychiatric disorders, including depression and PTSD (Yehuda, Giller, Southwick, Lowy, \& Mason, 1991; Young \& Korszun, 2010). The impact of stress and cortisol on emotion regulation - for example, the ability of cortisol to buffer mood or alter attentional biases (Het \& Wolf, 2007; Putman, Hermans, \& van Honk, 2010; Van Peer 
et al., 2007) — may be relevant to the pathophysiology of these disorders. It is thus of considerable potential interest to examine the impact of stress hormones like cortisol on emotion regulation processes. We therefore included placebocontrolled administration of exogenous hydrocortisone (HCT, which raises cortisol levels) in our design, to allow a preliminary examination of how cortisol might influence the impact of attention shifting and emotion labeling on both emotion expression and associated neural processes. Issue of timing and dosing are complex in cortisol administration studies. We elected to use a high dose and timing that would put measured cortisol levels in our subjects in a fairly stable, high stress range during completion of our tasks (Domes, Rothfischer, Reichwald, \& Hautzinger, 2005). We expected that this effort to mimic a sustained stressful situation would enhance implicit emotional responses and diminish any potential "emotion regulatory" effects of shifting attention away from the emotional stimuli.

\section{Method}

\section{Participants and procedures}

Forty individuals (20 females, 20 males; mean age $=22.8 \pm$ 5.4 years) were recruited from the local community and university populations. All were right-handed, healthy, unmedicated, and without psychiatric conditions, as confirmed by the Structured Clinical Interview for the DSM-IV. All provided IRB approved, written informed consent. They signed consent at a screening session, agreeing to random assignment to receive placebo or hydrocortisone (HCT), and they were informed about the fMRI task. They were also instructed to refrain from caffeine or heavy exercise prior to the experimental session. They reported to the fMRI laboratory for study between 11:00 a.m. and noon on a weekday, to control for diurnal variation in endogenous cortisol levels. They were assigned to placebo or HCT by constrained random assignment to insure equal sex representation (10 males, 10 females) in each group. Timing was structured to insure that subjects in the HCT group had reached peak cortisol prior to scanning and were in a window of stable high levels while completing the task (see below for details). Subjects read magazines while waiting.

\section{Stimuli and task}

Picture stimuli were presented in the scanner using E-Prime and a rear-projection screen (Psychological Software Tools, Inc., Pittsburgh, PA). Pictures were composite face/scene images comprised of 20 angry, 20 fearful, and 20 neutral expressions (Ekman \& Friesen, 1976; Gur et al., 2002) superimposed on 20 building scenes (10 indoor, 10 outdoor; Klumpp et al., 2012; Sripada et al., 2013). To localize the face and place processing brain areas, we used an additional 10 neutral faces and 10 indoor or outdoor scenes as noncomposite pictures. There were 80 unique gray-scale pictures in total.

The Shifted-Attention Emotion Appraisal Task (SEAT) is illustrated in Fig. 1a. This builds on prior work that used simple face pictures to study emotion labeling as an emotion regulation procedure (Chen, Welsh, Liberzon, \& Taylor, 2010), allowing study of both attention shifting and this type of appraisal in a single paradigm. Participants were shown our composite images and asked to respond to three different questions regarding each image: (1) pay attention to the face on the composite picture and determine if it is male or female (male/female condition); (2) pay attention to the scene on the composite picture, and determine if it is indoor or outdoor (indoor/outdoor condition); (3) pay attention to the face on the composite picture, and determine if you like or dislike the face (like/dislike condition). In all conditions the composite pictures displayed fearful, angry, or neutral faces. The male/female condition maintains attention on the emotional stimuli without engaging appraisal and is a standard fMRI approach to studying implicit emotional processing (FusarPoli et al., 2009). The other two conditions involve the same type of implicit emotional processing (emotional facial expressions are present and are processed), but additionally involve two types of nonintentional emotional regulation, namely (1) attention redirection (indoor/outdoor condition) and (2) appraisal (like/dislike condition). Each composite picture was presented three times, once in each condition, with condition type in random order (180 trials total). Correct responses in the male/female and indoor/outdoor conditions involved accurately identifying the sex of the face (male/female) or the location of the scene (indoor/outdoor). Noncomposite pictures (face or place only) were presented in 40 trials in which participants were simply asked to determine whether it was a face or place. A total of 220 trials were randomly ordered across four runs with 55 trials per run. Trials comprised a centered fixation crosshair for $\sim 3-8$ seconds, judgment cue for $750 \mathrm{~ms}$ $+250 \mathrm{~ms}$ blank screen, and then composite pictures for 1,500 ms. Prior to experimental trials, subjects completed a practice session with images not used in the experiment.

\section{Cortisol administration and salivary cortisol analysis}

HCT was administered as a single oral dose of $100 \mathrm{mg}$, 120 minutes before start of neuroimaging. Peak levels of cortisol occur approximately 1.2 hours after HCT administration and slowly decline over several hours (Derendorf et al., 1991). Saliva samples were collected at $-110,-90,-70,-50,-30,-10$, and +35 minutes relative to start of the task in the scanner. Samples were frozen at $-80{ }^{\circ} \mathrm{C}$ and thawed before being assayed in triplicate using commercially available Coat-aCount radioimmunoassay kits from Diagnostic Products 
(a)
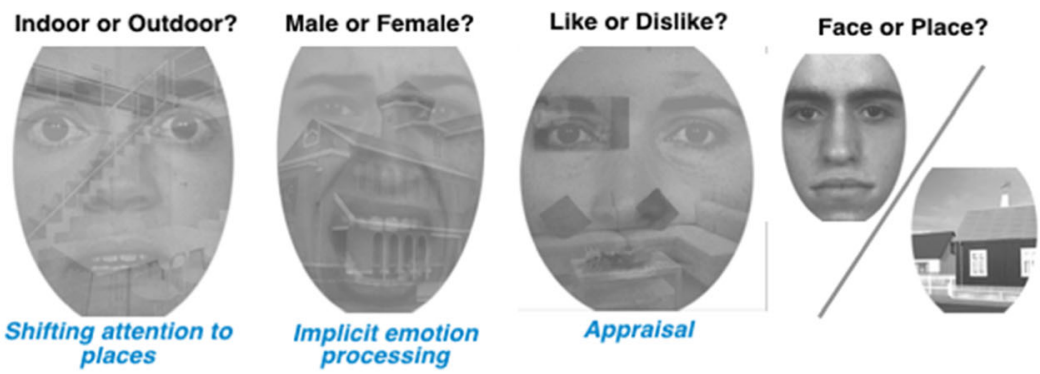

(b) Implicit emotion processing: Male/Female - Face

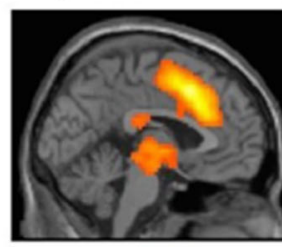

$x=-2$

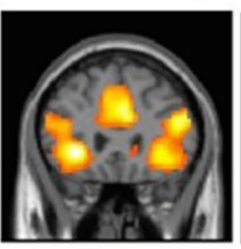

$\mathrm{y}=27$

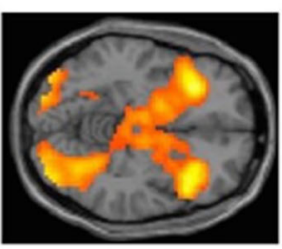

$z=-7$
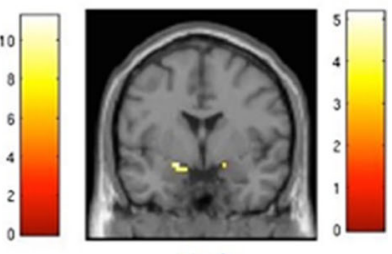

(c) Shifting Attention: Indoor/Outdoor - Male/Female

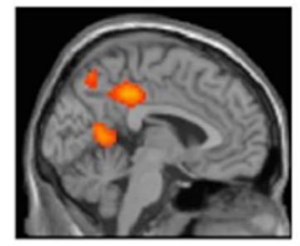

$\mathrm{x}=-3$

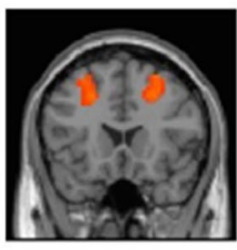

$y=20$

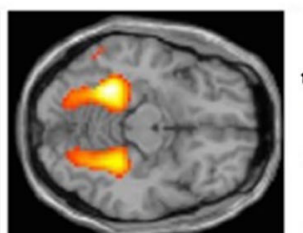

$z=-12$

\section{(d) Appraisal: Like/Dislike - Male/Female}

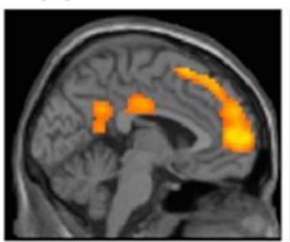

$x=-3$

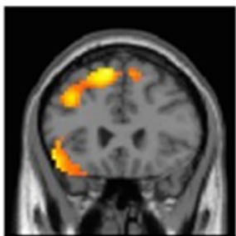

$\mathrm{y}=\mathbf{2 5}$

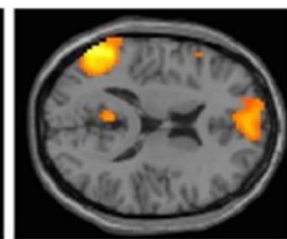

$z=16$

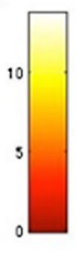

$y=-1$

Fig. 1 Task schematic and brain activation patterns for main contrasts. Sample stimuli and instructions/groups are presented in panel a and brain activity maps in panels b, $\mathbf{c}$, and $\mathbf{d}$. The left hemisphere is on the top in the axial slices, and on the left in the coronal slices (Color figure online)

Corporation (Los Angeles, CA). The intra- and interassay variabilities were less than $5 \%$ and $10 \%$, respectively.

Cortisol assay results were analyzed to document a significant rise in response to HCT administration, using SPSS 17.0 (Chicago, IL, U.S.A) and a three-way, mixed analysis of variance (ANOVA, $p<.05$ considered significant). Treatment (HCT, placebo) and participant sex (male, female) were between-subjects factors and time relative to administration $(-110-90,-70,-50,-30,-10$, and +35 minutes) was the within-subjects factor. Cortisol level (in ug/dl) was the dependent variable. Bonferroni correction was applied for post hoc pairwise comparisons.

\section{Behavioral analysis}

Behavioral data were analyzed using SPSS 17.0 (Chicago, IL, USA) with a mixed-effect general linear model (GLM). Treatment (HCT, placebo) and participant sex (male, female) served as between-subjects factors while task conditions (indoor/outdoor, male/female, like/dislike) and pictured facial emotion (angry, fearful, neutral) served as within-subjects factors. Reaction time (RT) was the primary dependent variable in this model. Accuracy was also analyzed as a continuous dependent variable, but since there was no "correct" response in the like/dislike condition, the task condition factor in this model had only two levels (indoor/outdoor and male/female). In the like/dislike condition, we also analyzed the number of likes and dislikes as a categorical dependent variable with treatment and sex groups as between-subjects factors and facial emotion as within-subjects factor.

\section{MRI acquisition and preprocessing}

All scanning was performed with blood-oxygen-leveldependent (BOLD) sensitive whole-brain fMRI on a 3.0 Tesla GE Signa System (General Electric; Milwaukee, WI) 
using a standard radiofrequency coil. A total of $760 \mathrm{~T} 2 *_{\text {- }}$ weighted reverse spiral gradient-recall echo volumes, with BOLD contrast (echo time $=30 \mathrm{~ms}$, repetition time $=$ $2,000 \mathrm{~ms}, 64 \times 64$ matrix, flip angle $=90$ degree, field of view $=22 \mathrm{~cm}, 40$ contiguous $3-\mathrm{mm}$ axial slices per volume), were acquired during a single session. A high-resolution T1 scan (3D-SPGR; $256 \times 160$ matrix, field of view $=24 \mathrm{~cm}$; slice thickness $=1.2 \mathrm{~mm}$ ) was also acquired for anatomical localization. The MRI images were preprocessed and analyzed using in-house batch mode of statistical parametric mapping (SPM8; Wellcome Trust Centre for Neuroimaging) provided by a Methods Core Team in the Department of Psychiatry at the University of Michigan. Slice timing correction was performed for functional volumes. Functional volumes were realigned to the first volume in the experiment to correct for head motion, co-registered with the high-resolution sagittal images, anatomically normalized to the Montreal Neurological Institute (MNI) template brain, resampled to $3 \times 3 \times 3 \mathrm{~mm}$ voxels, and smoothed with an $8 \times 8 \times 8$-mm kernel.

\section{MRI data analysis}

The preprocessed MRI data were analyzed using the general linear model framework in SPM8. There were 11 event regressors modeled: nine regressors for the composite pictures (three types of faces by three types of instruction) and two regressors for the noncomposite pictures (face only and place only). The onsets and durations of events were convolved with a canonical hemodynamic response function (HRF) to create the event regressors, in addition to covariates of six image realignment parameters to reduce movement induced artifacts. In the first-level analysis for each participant, the parameter estimates of event regressors were computed at each voxel. Appropriate linear contrasts were applied to the parameter estimates to produce contrast images and statistical parametric maps (SPM $t$ map). To evaluate activations associated with task, treatment, and sex effects, we've constructed a mixed ANOVA model to test the influence of the aforementioned variables. We then performed post-hoc tests for three task conditions - implicit emotion processing, emotion regulation by shifting attention, and emotion regulation by appraisal, respectively, by creating three contrasts ([male/female face-only], [indoor/outdoor - male/female] and [like/dislike - male/female]) - to characterize effects within regions from the main effect of task. Due to intrinsic limitation of the analytic package including all four factors in a single ANOVA model was not possible, so to further examine the effect of facial emotions on brain activations, we constructed a separate mixed ANOVA model that included emotion, treatment and sex instead of task. The contrast images of interest in these first-level models were used as subject-specific dependent variables in second-level random-effect models. Statistical threshold for whole-brain analysis were set at voxel-wise
FWE $p<.05$. In addition to whole brain analysis, we also created a mask from activations seen in the whole brain analysis of task main effects (voxel-wise FWE $p<.05$ ) for use in group analyses of HCT treatment and sex.

Specific regions of interests (ROIs) were also utilized, selected based on prior work (Sudheimer et al., 2013) in regards to implicit emotion processing (male/female condition) and derived from the Anatomical Automatic Labeling software (Tzourio-Mazoyer et al, 2002). These ROIs included amygdala, subgenual anterior cingulate cortex, and ventral medial prefrontal cortex (all small volume corrected at voxel-wise FWE $p<.05$ ). Beta values of surviving brain activations were extracted and analyzed with mixed ANOVA with significance threshold set at $p<.05$, Bonferroni corrected.

\section{Results}

\section{Behavioral results}

For reaction time (RT), there was a significant main effect of task, $F(2,72)=7.528, p=.001$, but no main effects of treatment, sex or facial emotion. The task effect was due to faster RTs for the indoor/outdoor condition compared to the male/ female condition, $t(238)=-3.042, p=.002$. There was also a significant Task $\times$ Facial emotion interaction, $F(4,144)=$ $13.788, p<.001$-responses were faster for angry faces compared to neutral faces in the like/dislike condition $\left(M_{\text {angry }}=\right.$ $1,029 \mathrm{~ms} ; M_{\text {fearful }}=1,084 \mathrm{~ms} ; M_{\text {neutral }}=1,155 \mathrm{~ms} ; p_{\text {angry-neutral }}$ $\left.<.02 ; p_{\text {fearful-neutral }} n s\right)$. There was also a significant Treatment $\times$ Facial Emotion interaction, $F(2,72)=3.626, p=.032$ - the HCT treated group responded faster to angry faces compared to neutral faces $\left(M_{\text {neutral }}=1,111 \mathrm{~ms} ; M_{\text {angry }}=1,068 \mathrm{~ms} ; p_{\text {angry- }}\right.$ neutral $<.05)$ with no difference between emotions for the placebo group $\left(M_{\text {neutral }}=1,071 \mathrm{~ms} ; M_{\text {angry }}=1,081 \mathrm{~ms} ; p_{\text {angry }}\right.$ neutral $n s)$.

Overall accuracy in the male/female and indoor/outdoor conditions was greater than $80 \%$ (well above chance but below the $100 \%$ ceiling). The GLM here revealed main effects of task, $F(1,36)=137.03, p<.001$, and emotion, $F(2,72)=$ $29.29, p<.001$, but no main effects of treatment or sex. Subjects were more accurate in the indoor/outdoor condition than the male/female condition $(89.0 \%$ vs. $74.8 \%, p<.05)$. Subjects were less accurate for angry or fearful facial emotions compared to neutral $\left(M_{\text {angry }}=79.8 \% ; M_{\text {fearful }}=79.6 \%\right.$; $\left.M_{\text {neutral }}=86.3 \% ; p_{\text {angry-neutral }}<.025 ; p_{\text {fearful-neutral }}<.025\right)$. This effect was primarily driven by reduced accuracy for angry and fearful faces in the male/female condition (significant Task $\times$ Facial Emotion interaction): $F(2,72)=8.97, p<.001$; $M_{\text {angry }}=71.8 \% ; M_{\text {fearful }}=71.0 \% ; M_{\text {neutral }}=81.6 \%$. There was also a significant three-way interaction for Task $\times$ Treatment $\times$ Sex, $F(1,36)=11.72, p=.0015$. In the male/female condition, HCT treated male subjects were significantly less accurate 
than HCT treated female subjects, $t(58)=-4.43, p<.001$; $M_{\text {male }}=66.0 \%, M_{\text {female }}=80.1 \%$, and displayed the numerically worst accuracy seen in any cell in the study (see Supplementary Table S1 for full accuracy and RT data).

The GLM on like and dislike judgements within the like/dislike condition revealed only a main effect of facial emotion, $F(2,74)=25.91, p<.001$. Angry and fearful faces were more disliked than neutral faces, $F(1,37)=27.42, p<$ .001 .

For salivary cortisol, there were main effects of treatment, $F(1,36)=62.39, p<.0001$, sex, $F(1,36)=23.82, p<.0001$, and time, $F(6,216)=15.62, p<.0001$. Cortisol levels rose dramatically over time in response to HCT, but not following placebo, and did so more in males than females (see Supplementary Material, S2, for graphical display). At time of first measurement, cortisol levels (-110 min) did not differ between treatment groups, $t(38)=0.10, n s$, or between sex groups $($ males $=0.164 \mathrm{ug} / \mathrm{dl} ;$ females $=0.117 \mathrm{ug} / \mathrm{dl}), t(38)$ $=2.23, n s)$. They became significantly elevated over initial values 70 minutes after HCT administration (-50 min relative to scanning), $t(38)=4.55, p=.00005$. They remained significantly higher with HCT than placebo during the fMRI session ( 3.17 vs. $0.080 \mathrm{ug} / \mathrm{dl})$. Males had significantly higher cortisol levels than females during the scanner task, $t(18)=7.75, p=$ $.0000003 ; 3.59 \pm 0.27$ vs. $1.22 \pm 0.15 \mathrm{ug} / \mathrm{dl}$. However, levels during the task were significantly higher than baseline for both males and females who received HCT, male: $t(18)=-12.796$, $p<.00001$; female: $t(18)=-7.321, p<.00001$. Task cortisol levels were not significantly elevated above baseline for either sex after placebo, male: $t(18)=1.467, n s ;$ female: $t(18)=$ $3.241, n s$.

\section{Functional MRI results}

\section{Main effects of task, treatment, sex, and emotion}

There was a main effect of task in our first mixed ANOVA model involving task, treatment and sex under whole brain family-wise threshold at voxel-wise FWE $p<$ .05 . There was no main effect of treatment or sex on brain activation in this model and the task by treatment interaction was not significant. We did find an interaction effect of treatment and sex that survived ROI analysis with a mask derived from activations from all tasks at whole brain family-wise threshold voxel-wise FWE $p<.05$. In our second mixed ANOVA model examining the main effects of emotions, treatment, and sex, we found no main effect of emotion, and we saw sex by treatment interaction present in the first ANOVA model. To further investigate task effects on brain activations, we performed post hoc comparisons and the peak activations are reported in the paragraphs below and Table 1 .
Effects of implicit emotion processing: male/female-face-only contrast

The contrast for implicit emotion processing was constructed by comparing the male/female condition over the face-only condition (in which all facial expressions were neutral). Whole brain analysis with family-wise error (FWE) $p<.05$ threshold revealed several significant clusters. Consistent with other implicit emotion activation studies (Fusar-Poli, 2009), a significant activation peak was found in left amygdala ([-18, $1,-11] ; Z=4.90 ; k=4 ; p<.001)$. Dorsal medial prefrontal cortex (dmPFC) and dorsal anterior cingulate cortex (dACC) were also consistently activated by emotional stimuli $([6,14$, 43]; $Z=6.94 ; k=552 ; p<.001)$. Activation was also seen in bilateral inferior frontal gyrus (IFG) and anterior insula (AI) clusters (left IFG/AI: [-33, 26, -2]; $Z=7.47 ; k=477 ; p<.001$; right IFG/AI: [39, 20, -5]; $Z=6.28 ; k=300 ; p<.001$ ). Bilateral occipital lobes were also activated (whole-brain results are summarized in Table 1 and Fig. 1b).

\section{Effects of shifting attention: indoor/outdoor-male/female contrast}

In the indoor/outdoor condition (as compared to the implicit emotion processing-male/female condition), there was a main effect of shifting attention in the left and right parahippocampal areas / fusiform gyrii ([-30, -43, -17]; $Z>$ $7.56 ; k=339 ; p<.001 ;$ and $[27,-46,-11] ; Z=6.90 ; k=235 ; p$ $<.001$, respectively) with overlapping areas in the parahippocampal place area (PPC). Additionally, there were significant peaks in left and right calcarine/precuneus/lingual gyrus (left: $[-15,-58,13] ; Z=7.28 ; k=198 ; p<.001$; right: $[18,-55,16] ; Z=7.55 ; k=189 ; p<.001)$. The bilateral occipital lobes were also significantly activated during shifting attention (left: $[-39,-82,31] ; Z=6.78 ; k=238 ; p<$ .001 ; right: $[45,-76,25] ; Z=7.56 ; k=163 ; p<.001)$. We saw no significant activations in emotion processing areas. In addition, significant activations in the PCC and dIPFC were also observed (PCC: $[-3,-37,40] ; Z=5.55 ; k=107 ; p<.001$; dIPFC: [33, 29, 40]; $Z=4.94 ; k=27 ; p=.001$; whole-brain results are summarized in Table 1 and Fig. 1c).

\section{Effects of appraisal modulation: like/dislike-male/female contrast}

Modulation effects of appraisal (like/dislike) as compared to the implicit emotion processing condition (male/female) were detected in several cortical areas. Regions in the frontal-parietal executive control networks, including dIPFC ([-36, 26, 37]; $Z=5.24 ; k=20 ; p=.002)$ and parietal lobe $([-54,-55,25] ; Z=7.04 ; k=388 ; p<$ $.001)$, were significantly activated. Furthermore, consistent with previous appraisal (Liberzon et al., 2000; 
Table 1 Brain activation associated with each contrast

\begin{tabular}{|c|c|c|c|c|c|c|}
\hline Region & Side & $Z$ & $k E$ & $x$ & $y$ & $z$ \\
\hline \multicolumn{7}{|c|}{ Implicit emotional processing-male/female-face-only contrast } \\
\hline Amygdala & $\mathrm{L}$ & 4.9 & 4 & -18 & -1 & -11 \\
\hline $\mathrm{dmPFC} / \mathrm{dACC}(\mathrm{BA} 6 / 8 / 9 / 32)$ & $\mathrm{L} / \mathrm{R}$ & 6.94 & 552 & 6 & 14 & 43 \\
\hline \multirow[t]{2}{*}{ Inferior frontal gyrus } & $\mathrm{L}$ & 7.47 & 340 & -33 & 26 & 2 \\
\hline & $\mathrm{R}$ & 6.71 & 374 & 51 & 23 & 22 \\
\hline \multirow{2}{*}{ Anterior insula } & $\mathrm{L}$ & 7.47 & 123 & -33 & 24 & -6 \\
\hline & $\mathrm{R}$ & 6.28 & 102 & 37 & 24 & -3 \\
\hline Cingulate gyrus (BA24) & $\mathrm{L} / \mathrm{R}$ & 6.94 & 219 & 6 & 23 & 34 \\
\hline \multirow[t]{2}{*}{ Fusiform } & $\mathrm{L}$ & 8.39 & 66 & -36 & -73 & -20 \\
\hline & $\mathrm{R}$ & 7.25 & 255 & 36 & -64 & -20 \\
\hline \multirow[t]{2}{*}{ Thalamus } & $\mathrm{L}$ & 4.7 & 12 & -9 & -16 & 5 \\
\hline & $\mathrm{R}$ & 4.86 & 14 & 9 & -13 & 4 \\
\hline \multirow[t]{2}{*}{ Occipital/lingual } & $\mathrm{L}$ & 5.78 & 157 & -30 & -79 & 19 \\
\hline & $\mathrm{R}$ & 6.87 & 558 & 33 & -91 & 4 \\
\hline \multirow[t]{2}{*}{ Parietal lobe } & $\mathrm{L}$ & 6.31 & 138 & -24 & -61 & 46 \\
\hline & $\mathrm{R}$ & 5.21 & 26 & 30 & -57 & 46 \\
\hline \multicolumn{7}{|c|}{ Shifting attention: Indoor/outdoor-male/female contrast } \\
\hline \multirow[t]{2}{*}{ Parahippocampus/fusiform } & $\mathrm{L}$ & $>8$ & 216 & -30 & -43 & -17 \\
\hline & $\mathrm{R}$ & 6.9 & 190 & 27 & -46 & -11 \\
\hline \multirow[t]{2}{*}{ Occipital } & $\mathrm{L}$ & 6.78 & 232 & -39 & -82 & 31 \\
\hline & $\mathrm{R}$ & 7.56 & 147 & 45 & -76 & 25 \\
\hline \multirow[t]{2}{*}{ Cuneus/precuneus/calcarine/lingual } & $\mathrm{L}$ & 7.76 & 166 & -15 & -58 & 13 \\
\hline & $\mathrm{R}$ & 7.55 & 163 & 18 & -55 & 16 \\
\hline Middle frontal gyrus & $\mathrm{R}$ & 4.94 & 27 & 33 & 29 & 40 \\
\hline PCC & $\mathrm{L} / \mathrm{R}$ & 5.55 & 94 & -3 & -37 & 40 \\
\hline \multicolumn{7}{|l|}{ Appraisal: Like/dislike-male/female contrast } \\
\hline Inferior frontal gyrus (BA44/45/47) & $\mathrm{L}$ & 7.04 & 32 & -54 & 23 & -5 \\
\hline mPFC (BA10/8/6)/DLPFC (BA9) & $\mathrm{L}$ & 6.47 & 339 & -9 & 38 & 46 \\
\hline Middle temporal/angular gyrus (BA39/40) & $\mathrm{L}$ & 7.04 & 297 & -54 & -55 & 25 \\
\hline PCC & $\mathrm{L} / \mathrm{R}$ & 5.6 & 21 & -9 & -49 & 28 \\
\hline dmPFC (BA10) & $\mathrm{L} / \mathrm{R}$ & 5.21 & 101 & -3 & 62 & 7 \\
\hline Middle frontal gyrus (BA9) & $\mathrm{L}$ & 5.24 & 20 & -36 & 26 & 37 \\
\hline \multicolumn{7}{|l|}{ Treatment $\times$ Sex interaction } \\
\hline dmPFC (BA10) & $\mathrm{L}$ & 4.42 & 4 & -3 & 20 & 55 \\
\hline
\end{tabular}

Mechias, Etkin, \& Kalisch, 2010; Phan, Taylor, Welsh, \& Ho, 2004) and explicit emotion regulation studies (McRae et al., 2010; Silvers, Weber, Wager, \& Ochsner, 2015) large areas in the dorsal and rostral $\mathrm{mPFC}$ were activated (dmPFC: [-9 38 46]; $Z=6.47 ; k=340 ; p<.001$; rmPFC: $[-3,62,7] ; Z=5.21 ; k=107 ; p<.001)$. Smaller cluster areas $(k<50)$ that were also significant include the left IFG ([-54, 23, -5]; $Z=5.70 ; k=43 ; p<.001)$ and left PCC ([-9, -49, 28]; $Z=5.60 ; k=31, p<.001)$, regions previously linked to volitional reappraisal (Goldin, McRae, Ramel, \& Gross, 2008; Ochsner et al., 2004;)whole-brain results are summarized in Table 1 and Fig. 1d).

\section{Cortisol effects}

As noted, fMRI mixed ANOVA revealed no significant main effect of treatment and no interaction between treatment and task, suggesting that HCT had no impact on the analyses reported above. In support of this conclusion, direct comparison of the HCT and placebo groups in parallel analyses across all tasks also revealed no significant brain activation differences between them (at either a whole brain or clusterwise FWE $p<$ .05 level). For further verification, we also repeated the main analyses on HCT and placebo groups separately. The brain activation patterns seen in the combined analyses were similarly present in each group by itself (both visually and in 
significance tests using a clusterwise threshold FWE $p<.05$; data available upon request).

Despite the lack of any main effects of cortisol or sex (no brain activation maps survived whole brain family-wise error (FWE) threshold $p<.05$ in implicit emotional activation, attention shifting, or appraisal conditions) on brain activation, cortisol effects did emerge in interaction with sex across the three tasks. HCT uniformly reduced activation (relative to placebo) in female subjects but increased activation (relative to placebo) for male subjects in the dmPFC $([-3,20,55] ; Z=$ $4.42 ; k=4 ; p<.001 ;$ Fig. 2). The differences in dmPFC activation were significant in ROI analysis at FWE $p<.05$ threshold using a mask derived from activations in all tasks (male/female, indoor/outdoor, and like/dislike; whole brain voxel-wise FWE $p<.05$ threshold) from all subjects.

We also examined activations in a priori regions that we found to be sensitive to cortisol administration in our previous study (Sudheimer et al., 2013), including amygdala, sgACC, and $\mathrm{vmPFC}$, in the context of implicit emotion processing (male/female condition). The extracted betas (from the implicit emotion task) were analyzed post hoc with a $2 \times 2$ ANOVA (Treatment $\times$ Sex) with Bonferroni corrections. In both the $\mathrm{vmPFC}$ and $\mathrm{sgACC}$, there was a significant Treatment $\times$ Sex interaction, vmPFC: $F(1,26)=4.1501, p<.05$; $\operatorname{sgACC}: F(1$, $36)=4.5953, p<.05$. Overall, we observed cortisol-induced increases in activations for the females but not for the males in all three regions, vmPFC: $t(58)=-3.7476, p<.005$; sgACC: $t(58)=-2.9658, p<.005$; amygdala: $t(58)=-2.4351, p<.02$ (Fig. 3).

\section{Discussion}

Our primary goal was to examine the neural effects of attention shifting and appraisal of emotion, to determine whether these cognitive processes effected responses to emotional stimuli via pathways parallel to those involved in volitional, explicitly instructed emotion regulation techniques, such as reappraisal (Ochsner, Bunge, Gross, \& Gabrieli, 2002; Gross \& Barrett, 2011). If so, they might be usefully further explored as implicit emotion regulation strategies. We were also interested in the effects of high dose cortisol on this circuitry, as a proxy for cognition-emotion interactions under high stress conditions. We used a novel task that allowed us to assess implicit emotional responses (male/female condition), attentional shift (indoor/outdoor condition), and appraisal (like/dislike condition). Behaviorally, shifting attention to house scenes (indoor/outdoor condition) increased accuracy and reduced reaction time as compared to attending to emotional faces. Negative emotional stimuli (angry and fearful faces) led to more "dislike" judgments and diminished performance accuracy as compared to neutral stimuli. As expected, implicit emotion
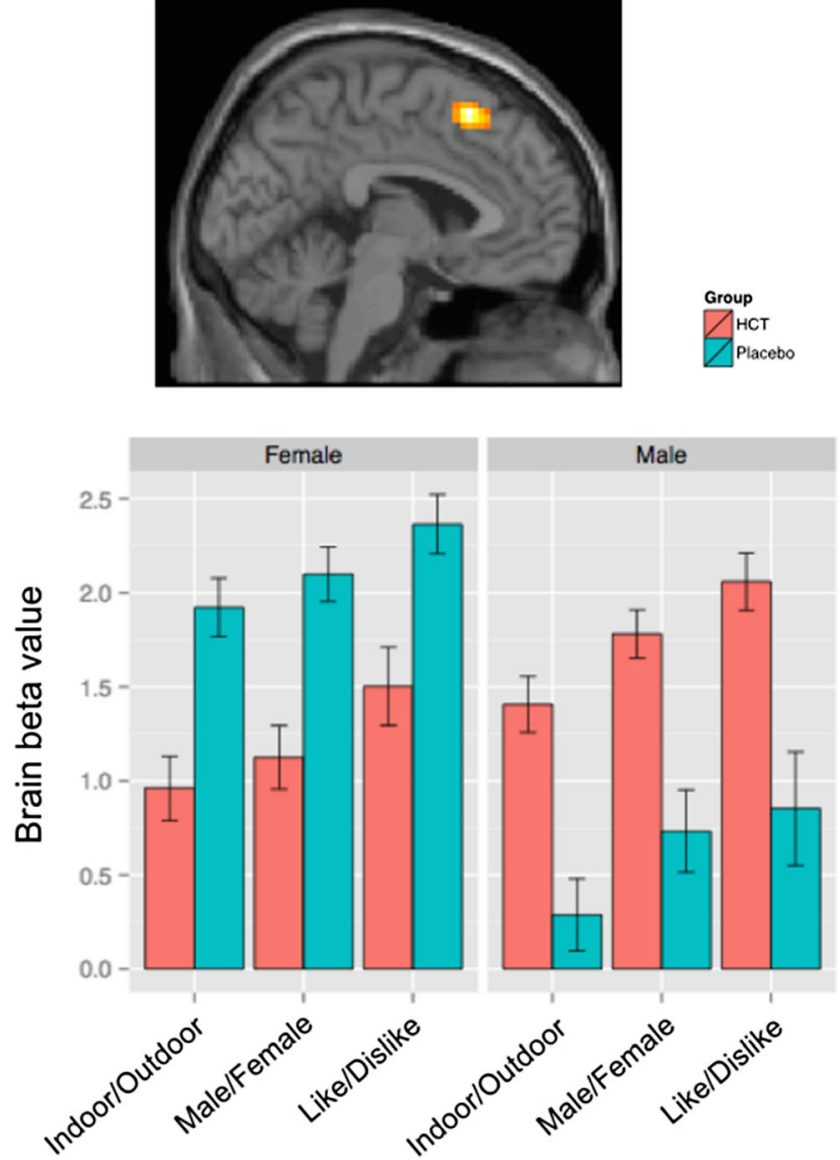

Fig. 2 Effects of hydrocortisone differed by subject sex. The top panel shows brain activations associated with the interaction of HCT and sex across all three tasks $(p<.001$, unc. for display). The only significant peak was in dmPFC $(-3,20,55)$. HCT reduced activation in females, relative to placebo, whereas it increased activation relative to placebo in males (Color figure online)

processing (attending to an emotional face, but to identify its sex) robustly activated amygdala, insula and $\mathrm{mPFC}$ regions. Attention shifting (attending to the building component of the compound images, rather than the faces) activated dIPFC and PCC, which are regions previously linked to emotional regulation (Gross \& Thompson, 2007). The attention shift also activated fusiform and parahippocampal gyri and bilateral occipital lobes. During appraisal of emotion (contrasting an emotion labeling condition to implicit emotional processing), large areas of dorsal to rostral $\mathrm{mPFC}$ were activated, consistent with prior work on emotion labeling as an emotion regulation strategy (Lieberman et al., 2007; Payer et al., 2012; Phan et al., 2004; S. F. Taylor et al., 2003). IFG, a region implicated in volitional emotion regulation (Kim et al., 2013), and portions of PCC, implicated in both appraisal and volitional emotion regulation (Liberzon et al., 2000; Phan et al., 2004), were activated as well. Single dose exogenous cortisol administration had no overall effect, but interacted with subject sex in modulating activation in the dmPFC area. 


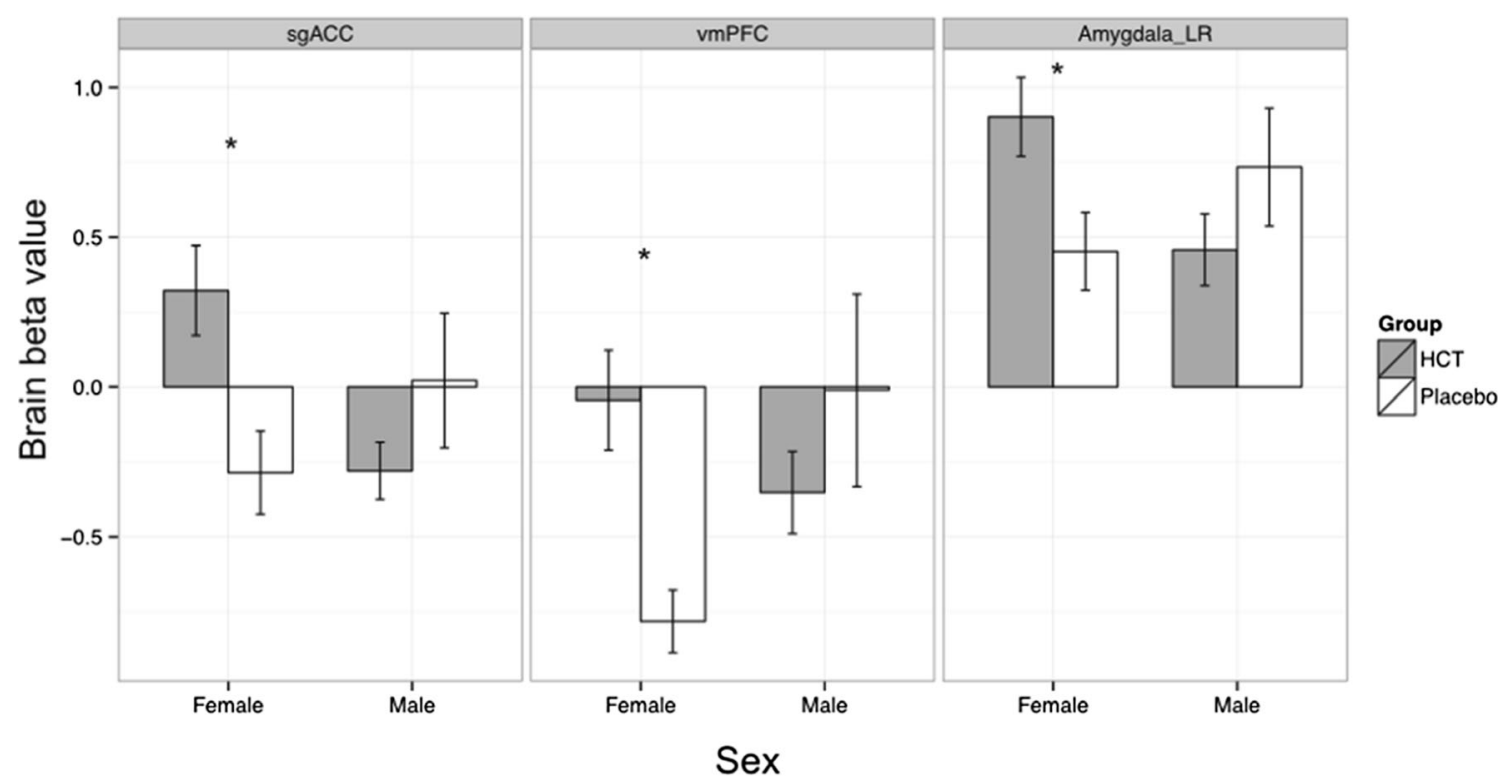

Fig. 3 Brain activations in a priori defined regions of interest, showing beta value extractions for males and females separately within each ROI, from the implicit emotion (male/female) task. Within each region, HCT produced increases in activation (or decreases in inactivation) that were not seen in males

In the implicit emotion processing condition, subjects were instructed to identify gender to focus their attention on the emotional faces, without explicitly directing attention to the emotional content. That content was nevertheless implicitly processed, as reflected in the increased "dislike" judgements, reduced gender identification accuracy, and lengthened reaction time for negative compared to neutral faces. We used this well-established implicit emotional processing condition (Breiter et al., 1996) as our comparator in order to avoid any explicit appraisal elements (e.g., naming emotions), in order to isolate appraisal processes (Critchley et al., 2000; George et al., 1993; Habel et al., 2007; Lane, Fink, Chau, \& Dolan, 1997) in the appraisal of emotion condition. As seen by others during processing of emotional faces (Adolphs, 2002; LeDoux, 1995; Schneider et al., 1997; Schneider, Gur, Gur, \& Muenz, 1994) and processing negative emotions more generally (Etkin, Egner, \& Kalisch, 2011; Maier et al., 2012), during implicit emotional processing our subjects activated amygdala and insula, as well as dorsal medial prefrontal cortex $(\mathrm{dmPFC})$ and the anterior portion of dorsal ACC (adACC).

Our task was designed to allow us to examine two common cognitive processes of daily life - shifting attention and appraisal of emotion - both of which can potentially modulate emotional responding and perhaps serve as emotion regulation strategies. Shifting attention in this context does not mean ignoring the stimuli altogether, but rather focusing volitional attention on components without emotional salience. This is analogous to a physician in an emergency room purposefully focusing attention on specific components of the injury, to assess and perhaps treat it, rather than allowing attention to be captured by the patient's distress and suffering. This likely modulates emotional responding in a way that permits more effective action. We expected that engagement of regions in the attention network would be required for this task, and that this would lead to decreased processing in emotion generating regions, which would parallel findings from volitional emotion regulation strategies (McRae et al., 2010) and support the idea that attention shifting can function as an emotion regulation process. We did in fact detect the expected activations in attention network components, while no activations were seen in emotion processing regions, in contrast to what was observed in the implicit emotion condition. Attention networks are thought to be comprised of alerting, orientating and execution/conflict monitoring components (Posner \& Petersen, 1990). During our shifting attention condition, we observed activation of lateral prefrontal cortex, the same region implicated in Posner's "orienting attention" network (Koenigsberg et al., 2010). We also observed activation in PCC. Activation in this region has been linked to target detection in a selective attention task (Shulman et al., 2010). Interestingly, PCC and precuneus activations have also been reported during emotional distancing, a volitional emotion regulation strategy (Koenigsberg et al., 2010), but not in distraction studies (Kanske, Heissler, Schönfelder, Bongers, \& Wessa, 2011; McRae et al., 2010). This suggests a potentially similar role for PCC in regulating some aspects of attention during specific types of emotion regulation, like shifting attention and distancing. Future studies will be required to further clarify the precise role of PCC in these processes. Shifting attention also activated fusiform gyrus and parahippocampal gyrus, with more overlapping areas in the parahippocampal 
place area (PPA; Epstein \& Kanwisher, 1998). Since subjects were instructed to shift attention to a place image (identifying it as inside or outside), increased activation in the parahippocampal place area is entirely consistent with the task and confirms that the manipulation worked, and subjects were focusing on the place component of the picture, as instructed. In summary, the attention shift condition activated attention related regions (dlPFC and PCC) and visual/space processing regions that were appropriate to the content of the stimuli to which attention was shifted, and this was associated with diminished activation in the emotion processing regions that had been activated in the implicit emotional activation condition.

We were specifically interested in regulation by appraisal of emotion, because appraisal is both a very common mechanism that is engaged automatically while processing emotional stimuli (Critchley et al., 2000; S. F. Taylor et al., 2003), and also can be used volitionally, as often practiced in cognitive and cognitive-behavioral approaches (Beck, Emery, \& Greenberg, 1985; Clark, 1986; Norton, Asmundson, Cox, \& Norton, 2000). In previous studies, both our lab (S. F. Taylor et al., 2003) and others (Hariri et al., 2003) have demonstrated that $\mathrm{mPFC} / \mathrm{ACC}$ regions are involved in appraisal and that engaging appraisal diminishes the emotional impact of negative stimuli (S. F. Taylor et al., 2003). Medial prefrontal cortex activations have been reported in the context of volitional emotion regulation tasks, such as reappraisal (Kalisch, 2009), and mPFC has been hypothesized to provide topdown control to emotion processing areas, such as the amygdala (Etkin et al., 2011). In contrast to reappraisal, our appraisal task did not involve a purposeful effort to modulate emotional responses; it simply asked subjects to identify one of their emotional responses to the face presented (as like or dislike). However, the large dorsal to rostral mPFC activations seen in our study overlap with the activations reported in studies of reappraisal (Ochsner et al., 2002; Phan et al., 2004), suggesting that a process that does not involve instructed, volitional modulation of emotion activated regulatory regions similar to those activated by purposeful regulation strategies. Rostral $\mathrm{mPFC}$ regions have been also implicated in processing of self and self-relatedness (Chiao et al., 2009; Gilbert et al., 2007; Phan et al., 2004), so the large rostral mPFC activations we saw may have also been partly elicited by the fact that in our appraisal task, subjects had to reflect on their own, internal emotional states. The dorsal lateral prefrontal cortex (dlPFC) activations that we saw also parallel the dIPFC activations seen in studies using volitional emotion regulation strategies such as reappraisal (Kim et al., 2013; Ochsner et al., 2002), again supporting the hypothesis that both volitional and implicit emotion regulation processes use shared neural networks to modulate emotional experience or expression.

We administered exogenous cortisol in a double blind fashion in order to examine effects of elevated stress hormone on appraisal and attention regulation. Cortisol has important cognitive and emotional effects on brain with high relevance to PTSD (Kaouane et al., 2012) and there is intriguing evidence of a potentially important role in PTSD treatment, likely mediated by these brain effects (Yehuda et al., 2015). Deeper understanding of its effects on all types of potential emotion regulation processes will be important in efforts to better understand disorders with dysregulated HPA axis function and determine how to optimally use cortisol therapeutically. Behaviorally, cortisol administration affected both reaction time and accuracy. It shortened reaction time to negative affective stimuli (angry faces) compared to neutral ones, while reducing accuracy during implicit emotion processing (male/female condition) specifically in male subjects. However, cortisol had no significant main effects on brain activation maps in fMRI analyses for any of the three tasks, and had no measurable impact in shaping the brain activation patterns seen in attention shifting and appraisal of emotions. On the other hand, there were significant sex differences in cortisol's brain effects across the tasks. Cortisol administration reduced activation in dorsal medial prefrontal cortex (dmPFC) in all three tasks in females, but it enhanced dmPFC activation in these tasks in males. Simultaneously, in females during the implicit emotion task it increased activation in sgACC, vmPFC, and amygdala, emotion processing regions previously shown to be sensitive to exogenous cortisol (Sudheimer et al., 2013). It did not have this effect in males.

Unfortunately, males and females also differed markedly in salivary cortisol levels achieved during the tasks. As a result, we cannot determine whether the neural differences between males and females reflect a true sex difference in brain effects of cortisol or are due to "dose" effects. They are of interest in either case (see below), but follow-up work will be needed to determine which explanation is correct. The sex differences in measured cortisol levels could reflect sex differences in cortisol binding globulin, which can differ between males and females, partly due to the influence of estrogen, which elevates $C B G$, leading to increased cortisol binding and reduced levels of free cortisol. Saliva measures reflect free cortisol, which is the active component most relevant to effects on brain (Hellhammer, Wüst, \& Kudielka, 2009). Free cortisol can be reduced in females by menstrual cycle effects on estrogen levels and by estrogen containing birth control pills (BCPs), neither of which were controlled here. Future studies will need to measure $\mathrm{CBG}$ and carefully control for cycle effects and BCP use in order to test for true sex differences in brain effects of cortisol and distinguish them from "dose" effects.

Glucocorticoids do have well-established dose-dependent effects on brain, with a dose-response curve that is often described as an inverted $U$. Enhanced cognitive function in different memory paradigms is often seen at "moderate" cortisol levels, with less optimal function at very low levels and deficits appearing at very high levels (Domes et al., 2005; Salehi, 
Cordero, \& Sandi, 2010; Schilling et al., 2013). We cannot determine whether the sexually dimorphic brain activations seen here represent "negative" effects of high cortisol in males in this paradigm, but the cortisol dose-response literature would certainly predict differential brain activity with moderate versus high free cortisol levels. The levels seen during our task in females would be considered comparable to moderate stress levels, whereas the levels seen in the males are only seen under conditions of high stress that perhaps includes physiological stress. The differential dose effects may be due to changing ratios of occupancy of mineralocorticoid (MR) and glucocorticoid (GR) receptors, with high occupancy of GR reducing the MR/GR ratio and producing detrimental effects (Bohbot, Gupta, Banner, \& Dahmani, 2011). Our male subjects who received HCT did display reduced accuracy in identifying faces as male or female, relative to all other groups, and this could reflect a detrimental effect of the uniquely high cortisol levels seen by their brains. If so, this would suggest that the inverted $U$ dose-response pattern for cortisol effects on memory, which has been the focus of most work in this area to date, may also apply to other cognitive functions. This accuracy deficit, however, is unlikely to be related to the increased dmPFC activity seen in these male subjects. The increased activity associated with moderate cortisol elevations seen in females in emotion processing regions (sgACC, vmPFC, and amygdala) could reflect an enhanced "appropriate" response during the implicit emotion task in which it was seen (this enhancement was one of our a priori expectations). The absence of this effect in males may reflect a "deficit" due to their very high cortisol levels. However, this is clearly highly speculative, and more work is definitely needed to explore the interesting potential explanations for and implications of these findings.

We also need to consider the possibility that the male-female differences in cortisol effects on dmPFC, sgACC, vmPFC, and amygdala were not solely due to different cortisol levels, but could also be influenced by sexually dimorphic brain sensitivities to cortisol within the brain. Others have reported sex differences in dmPFC during emotion perception (Hofer et al., 2006), but stress or cortisol effects were not "in play" in that study. Amygdala and vmPFC are rich in glucocorticoid receptors (Holsboer, 2000) and important in emotion processing and emotion regulation. If there are male-female differences in the sensitivity of these regions to free cortisol, these will be important to identify, as they could shape sexually dimorphic effects of cortisol on emotion regualtion strategies and capacities. Here, cortisol enhanced MPFC activation in men, perhaps supporting appraisal, while in women cortisol increased activation in other cortical regions - such as sgACC and $\mathrm{VmPFC}$ - that are involved in mood regulation and extinction retention. Though purely speculative at this point, there is clear potential relevance to well-known sex differencs in psychiatric disorders such as depression and PTSD, where
HPA axis dysregulation is also well established. More work is clearly needed.

\section{Limitations}

Several limitations must be acknowledged. We used emotional facial expressions as emotion generating stimuli. While this method has been widely used in fMRI studies (Fusar-Poli et al., 2009; Gur et al., 2002), it may have elicited less intense emotional responses than use of other emotion generating stimuli. Furthermore, in the implicit emotion condition, identifying the gender of the face allows the emotional content to be processed implicitly, but the identification task itself requires cognitive processing, and this cognitive processing could reduce emotional activation, so full extent of potential emotional activation may not have been seen even in this "baseline" condition. The cognitive work of identification could also potentially interact with appraisal or attention shifting strategies. We decided, however, that the benefits of engaging our subjects in a specific task and maintaining their attention outweighed the potential "losses" due to a weaker emotional activation signal or the potential for "subtracting out" regions involved in both appraisal and gender recognition.

It is also important to acknowledge that though we have use the terminology implicit emotion regulation to describe what is happening with attention shifting and emotion labeling activity, the concept of implicitness is complex and potentially controversial. We do see brain changes suggesting that emotion regulation areas of the brain are "in play" with these manipulations, but we could also simply call this "uninstructed" emotion regulation. Our main goal in using the word implicit is to differentiate these strategies from the explicit, volitional efforts to alter emotional experience that have been used in appraisal and related studies. We also note that mechanisms other than implicit emotion regulation could be shaping the results seen. For example, in the attention shift task, subjects could have been aware of interference with efforts to focus on the building created by the "pull" for attention from the embedded face. They may have resolved this interference by using "explicit" regulation strategies. Creative future work will be needed if we are to differentiate the explicit cognitive work of directing attention away from an emotionally evocative cue from what we have called implicit emotional regulation. We suspect that they use similar neural pathways and both can result in reduced emotional processing, so the differences may be mainly semantic, but work on this is needed.

Results from the cortisol infusion must be considered preliminary. As noted above, because males and females achieved very different free cortisol levels in response to the same dose of HCT, we cannot disentangle "dose" effects from sex differences in brain sensitivities. Substantially larger studies will be needed to fully dissect potential interactions 
between sex, cortisol levels, and cortisol effects on brain during emotion regulation processes. The between-group design used also has some drawbacks. It is less sensitive than a within subject design, which reduces some variability; and individual differences in cortisol response can contribute additional variance. However, we wanted to avoid repetition and learning effects that could pose substantive confounds (Wirth, Scherer, Hoks, \& Abercrombie, 2011). Use of exogenous cortisol is a simplified downstream proxy for actual stress. Cortisol is a known cognitive modulator, with established dysregulation in psychiatric disorders, so isolating its independent effects is of interest, but future studies will need to examine the effects of ecological stress, with its additional neural and psychosocial components, in these types of paradigms. Exogenous administration here was complicated by sex differences in levels achieved and high variability in levels among the males. We have seen this in other studies, and it needs clarification in follow-up work. Future studies will also need to examine impacts on other systems, such as the reward system, that are also affected by stress and cortisol.

\section{Conclusions}

Emotion regulation pathways have been the focus of recent studies, but most work has examined instructed, explicit, or "exogenous" regulation techniques (Kuhn et al., 2014). There has been debate in the literature as to whether all emotion regulation strategies must involve active, volitional efforts to change emotions (Gross \& Barrett, 2011). Here, we demonstrate the utility of a task that allowed us to explore cognitive processes that are not specifically targeting emotional processes or circuitry. We used this task to test their ability to alter activity in brain regions that are activated by more traditional emotion regulation techniques - by shifting attention or appraising an internal emotional state, without attempting intentionally to alter emotional responses. Attention shifting activated attention network brain areas (e.g., IPFC and PCC) and appropriate task-specific areas (e.g., parahippocampal place area, reflecting successful allocation of attention to the place component of the compound picture). Appraisal robustly activated $\mathrm{mPFC}$ as well as dIPFC and IFG, as also seen in instructed reappraisal strategies. In both, regions activated by implicit emotion processing were "quiet." The data suggest that both attention shifting and appraisal of emotions might be thought of as implicit emotion regulation strategies, acting through some of the same brain circuits involved in instructed emotion regulation. Further examination of such implicit emotion regulation processes appears warranted, with potential relevance to psychiatric disorders such as PTSD and social anxiety disorder, where emotion regulation processes and attentional control have clinical salience.

The sexually dimorphic brain responses to cortisol are intriguing despite the confound between "dose" and sex.
Understanding potential dose-dependent effects of cortisol on emotion regulation capacities is of clinical relevance to psychiatric disorders. It will be critical in efforts to determine the true role of the HPA axis in these disorders and the true potential utility of HPA axis interventions in treating them. Understanding sex differences in brain sensitivity to cortisol, if they exist, could help explain differences in emotion regulation strategies between men and women, and contribute to our understanding of sex differences in vulnerability to disorders like PTSD and depression. Disentangling "dose" and sex effects, which may have both been in play in this study, is essential in future work.

Acknowledgements The research reported in this article was supported by a grant from the National Institute of Mental Health (R24 MH075999) to I. Liberzon.

\section{Compliance with ethical standards}

Conflict of interest None

\section{Reference}

Adolphs, R. (2002). Neural systems for recognizing emotion. Current Opinions in Neurobiology, 12(2), 169-177.

Almeida, O. P., MacLeod, C., Ford, A., Grafton, B., Hirani, V., Glance, D., \& Holmes, E. (2014). Cognitive bias modification to prevent depression (COPE): Study protocol for a randomised controlled trial. Trials, 15, 282.

Anderson, A. K., Christoff, K., Panitz, D., De Rosa, E., \& Gabrieli, J. D. (2003). Neural correlates of the automatic processing of threat facial signals. Journal of Neuroscience, 23(13), 5627-5633.

Aron, A. R., Robbins, T. W., \& Poldrack, R. A. (2004). Inhibition and the right inferior frontal cortex. Trends in Cognitive Science, 8(4), 170-177.

Beck, A., Emery, G., \& Greenberg, R. (1985). Anxiety disorders and phobias: A cognitive approach. New York, NY: Basic.

Berking, M., \& Wupperman, P. (2012). Emotion regulation and mental health: Recent findings, current challenges, and future directions. Current Opinion in Psychiatry, 25(2), 128-134.

Bishop, S. J. (2008). Neural mechanisms underlying selective attention to threat. Annals of the New York Academy of Sciences, 1129, 141-152.

Bohbot, V. D., Gupta, M., Banner, H., \& Dahmani, L. (2011). Caudate nucleus-dependent response strategies in a virtual navigation task are associated with lower basal cortisol and impaired episodic memory. Neurobiology of Learning and Memory, 96(2), 173-180.

Breiter, H. C., Etcoff, N. L., Whalen, P. J., Kennedy, W. A., Rauch, S. L., Buckner, R. L.,...Rosen B. R. (1996). Response and habituation of the human amygdala during visual processing of facial expression. Neuron, 17, 875-887

Britton, J. C., Bar-Haim, Y., Clementi, M. A., Sankin, L. S., Chen, G., Shechner, T.,...Pine, D. S. (2013). Training-associated changes and stability of attention bias in youth: Implications for Attention Bias Modification Treatment for pediatric anxiety. Developmental Cognitive Neuroscience, 4, 52-64.

Britton, J. C., Lissek, S., Grillon, C., Norcross, M. A., \& Pine, D. S. (2011). Development of anxiety: The role of threat appraisal and fear learning. Depression and Anxiety, 28(1), 5-17. 
Britton, J. C., Suway, J. G., Clementi, M. A., Fox, N. A., Pine, D. S., \& Bar-Haim, Y. (2014). Neural changes with attention bias modification for anxiety: A randomized trial. Social Cognitive and Affective Neuroscience, 10(7), 913-920.

Browning, M., Holmes, E. A., Murphy, S. E., Goodwin, G. M., \& Harmer, C. J. (2010). Lateral prefrontal cortex mediates the cognitive modification of attentional bias. Biological Psychiatry, 67(10), 919-925.

Buchanan, T. W., \& Lovallo, W. R. (2001). Enhanced memory for emotional material following stress-level cortisol treatment in humans. Psychoneuroendocrinology, 26(3), 307-317.

Chen, A. C., Welsh, R. C., Liberzon, I., \& Taylor, S. F. (2010). 'Do I like this person?' A network analysis of midline cortex during a social preference task. NeuroImage, 51(2), 930-939.

Chiao, J. Y., Harada, T., Komeda, H., Li, Z., Mano, Y., Saito, D.,... Iidaka, T. (2009). Neural basis of individualistic and collectivistic views of self. Human Brain Mapping, 30(November 2008), 2813-2820.

Clark, D. M. (1986). A cognitive approach to panic. Behaviour Research and Therapy, 24(4), 461-470.

Critchley, H., Daly, E., Phillips, M., Brammer, M., Bullmore, E., Williams, S.,...Murphy, D. (2000). Explicit and implicit neural mechanisms for processing of social information from facial expressions: A functional magnetic resonance imaging study. Human Brain Mapping, 9(2), 93-105.

De Quervain, D. J., Aerni, A., Schelling, G., \& Roozendaal, B. (2009). Glucocorticoids and the regulation of memory in health and disease. Frontiers in Neuroendocrinology, 30(3), 358-370.

Derendorf, H., Mollmann, H., Barth, J., Mollmann, C., Tunn, S., \& Krieg, M. (1991). Pharmacokinetics and oral bioavailability of hydrocortisone. Journal of Clinical Pharmacology, 31(5), 473-476.

Domes, G., Rothfischer, J., Reichwald, U., \& Hautzinger, M. (2005). Inverted-U function between salivary cortisol and retrieval of verbal memory after hydrocortisone treatment. Behaviour Neuroscience, $119(2), 512-517$.

Eisenberg, N. (2000). Emotion, regulation, and moral development. Annual Review of Psychology, 51, 665-697.

Ekman, P., \& Friesen, W. V. (1976). Pictures of facial affect. Palo Alto, CA: Consulting Psychologists Press.

Elsesser, K., Freyth, C., Lohrmann, T., \& Sartory, G. (2009). Dysfunctional cognitive appraisal and psychophysiological reactivity in acute stress disorder. Journal of Anxiety Disorders, 23(7), 979-985.

Epstein, R., \& Kanwisher, N. (1998). A cortical representation of the local visual environment. Nature, 392(6676), 598-601.

Etkin, A., Egner, T., \& Kalisch, R. (2011). Emotional processing in anterior cingulate and medial prefrontal cortex. Trends in Cognitive Sciences, 15(2), 85-93.

Fusar-Poli, P., Placentino, A., Carletti, F., Landi, P., Allen, P., Surguladze, S.,...Politi, P. (2009). Functional atlas of emotional faces processing: A voxel-based meta-analysis of 105 functional magnetic resonance imaging studies. Journal of Psychiatry Neuroscience, 34(6), 418-432.

George, M. S., Ketter, T. A., Gill, D. S., Haxby, J. V., Ungerleider, L. G., Herscovitch, P., \& Post, R. M. (1993). Brain regions involved in recognizing facial emotion or identity: An oxygen-15 PET study. Journal of Neuropsychiatry and Clinical Neuroscience, 5(4), 384-394.

Gilbert, S. J., Williamson, I. D. M., Dumontheil, I., Simons, J. S., Frith, C. D., \& Burgess, P. W. (2007). Distinct regions of medial rostral prefrontal cortex supporting social and nonsocial functions. Social Cognitive and Affective Neuroscience, 2, 217-226.

Goldin, P. R., McRae, K., Ramel, W., \& Gross, J. J. (2008). The neural bases of emotion regulation: Reappraisal and suppression of negative emotion. Biological Psychiatry, 63(6), 577-586.

Gross, J. J. (1998). The emerging field of emotion regulation: An integrative review. Review of General Psychology, 2(3), 271.
Gross, J. J., \& Barrett, L. F. (2011). Emotion Generation and Emotion regulation: One or two depends on your point of view. Emotion Review, 3, 8-16.

Gross, J. J., \& Munoz, R. F. (1995). Emotion regulation and mentalhealth. Clinical Psychology-Science and Practice, 2(2), 151-164.

Gross, J. J., \& Thompson, R. A. (2007). Emotion regulation: Conceptual foundations. Handbook of Emotion Regulation, 3, 24.

Gur, R. C., Schroeder, L., Turner, T., McGrath, C., Chan, R. M., Turetsky, B. I.,...Gur, R. E. (2002). Brain activation during facial emotion processing. NeuroImage, 16(3, Pt. 1), 651-662.

Habel, U., Windischberger, C., Derntl, B., Robinson, S., Kryspin-Exner, I., Gur, R. C., \& Moser, E. (2007). Amygdala activation and facial expressions: Explicit emotion discrimination versus implicit emotion processing. Neuropsychologia, 45(10), 2369-2377.

Hannesdottir, D. K., \& Ollendick, T. H. (2007). The role of emotion regulation in the treatment of child anxiety disorders. Clinical Child and Family Psychology Review, 10(3), 275-293.

Hariri, A. R., Bookheimer, S. Y., \& Mazziotta, J. C. (2000). Modulating emotional responses: Effects of a neocortical network on the limbic system. Neuroreport, 11(1), 43-48.

Hariri, A. R., Mattay, V. S., Tessitore, A., Fera, F., \& Weinberger, D. R. (2003). Neocortical modulation of the amygdala response to fearful stimuli. Biological Psychiatry, 53(6), 494-501.

Hellhammer, D. H., Wüst, S., \& Kudielka, B. M. (2009). Salivary cortisol as a biomarker in stress research. Psychoneuroendocrinology, 34(2), 163-171.

Henckens, M. J., van Wingen, G. A., Joels, M., \& Fernandez, G. (2010). Time-dependent effects of corticosteroids on human amygdala processing. Journal of Neuroscience, 30(38), 12725-12732.

Het, S., \& Wolf, O. T. (2007). Mood changes in response to psychosocial stress in healthy young women: Effects of pretreatment with cortisol. Behavior Neuroscience, 121, 11-20.

Hofer, A., Siedentopf, C. M., Ischebeck, A., Rettenbacher, M. A., Verius, M.,...Fleischhacker, W. W. (2006). Gender differences in regional cerebral activity during the perception of emotion: A functional MRI study. NeuroImage, 32(2), 854-862.

Holsboer, F. (2000). The corticosteroid receptor hypothesis of depression. Neuropsychopharmacology, 23, 477-501.

Kalisch, R. (2009). The functional neuroanatomy of reappraisal: Time matters. Neuroscience \& Biobehavioral Reviews, 33(8), 1215-1226.

Kanske, P., Heissler, J., Schönfelder, S., Bongers, A., \& Wessa, M. (2011). How to regulate emotion? Neural networks for reappraisal and distraction. Cerebral Cortex, 21, 1379-1388.

Kaouane, N., Porte, Y., Vallée, M., Brayda-Bruno, L., Mons, N., Calandreau, L.,...Desmedt, A. (2012). Glucocorticoids can induce PTSD-like memory impairments in mice. Science, 335(6075), $1510-1513$.

Kim, P., Evans, G. W., Angstadt, M., Ho, S. S., Sripada, C. S., Swain, J. E.,...Phan, K. L. (2013). Effects of childhood poverty and chronic stress on emotion regulatory brain function in adulthood. Proceedings of the National Academies of Science of the United States of America.

Klumpp, H., Angstadt, M., \& Phan, K. L. (2012). Shifting the focus of attention modulates amygdala and anterior cingulate cortex reactivity to emotional faces. Neuroscience Letters, 514(2), 210-213.

Koenigsberg, H. W., Fan, J., Ochsner, K. N., Liu, X., Guise, K., Pizzarello, S.,... Siever, L. J. (2010). Neural correlates of using distancing to regulate emotional responses to social situations. Neuropsychologia, 48(6), 1813-1822.

Kompus, K., Hugdahl, K., Ohman, A., Marklund, P., \& Nyberg, L. (2009). Distinct control networks for cognition and emotion in the prefrontal cortex. Neuroscience Letters, 467(2), 76-80.

Kuhn, S., Haggard, P., \& Brass, M. (2014). Differences between endogenous and exogenous emotion inhibition in the human brain. Brain Structure and Function, 219(3), 1129-1138. 
Lane, R. D., Fink, G. R., Chau, P. M., \& Dolan, R. J. (1997). Neural activation during selective attention to subjective emotional responses. Neuroreport, 8(18), 3969-3972.

LeDoux, J. E. (1995). Emotion: Clues from the brain. Annual Review of Psychology, 46(1), 209-235.

Liberzon, I., Taylor, S. F., Fig, L. M., Decker, L. R., Koeppe, R. A., \& Minoshima, S. (2000). Limbic activation and psychophysiologic responses to aversive visual stimuli: Interaction with cognitive task. Neuropsychopharmacology, 23(5), 508-516.

Lieberman, M. D., Eisenberger, N. I., Crockett, M. J., Tom, S. M., Pfeifer, J. H., \& Way, B. M. (2007). Putting feelings into words: Affect labeling disrupts amygdala activity in response to affective stimuli. Psychological Science, 18(5), 421-428.

Maier, S., Szalkowski, A., Kamphausen, S., Perlov, E., Feige, B., Blechert, J.,...Tüscher, O. (2012). Clarifying the role of the rostral $\mathrm{dmPFC} / \mathrm{dACC}$ in fear/anxiety: Learning, appraisal or expression? PLOS ONE, 7(11). doi:10.1371/journal.pone.0050120

McRae, K., Hughes, B., Chopra, S., Gabrieli, J. D. E., Gross, J. J., \& Ochsner, K. N. (2010). The neural bases of distraction and reappraisal. Journal of Cognitive Neuroscience, 22, 248-262.

Mechias, M. L., Etkin, A., \& Kalisch, R. (2010). A meta-analysis of instructed fear studies: Implications for conscious appraisal of threat. NeuroImage, 49(2), 1760-1768.

Norton, P. J., Asmundson, G. J., Cox, B. J., \& Norton, G. R. (2000). Future directions in anxiety disorders: Profiles and perspectives of leading contributors. Journal of Anxiety Disorders, 14(1), 69-95.

Ochsner, K. N., Bunge, S. A., Gross, J. J., \& Gabrieli, J. D. (2002). Rethinking feelings: An FMRI study of the cognitive regulation of emotion. Journal of Cognitive Neuroscience, 14(8), 1215-1229.

Ochsner, K. N., \& Gross, J. J. (2007). The neural architecture of emotion regulation. Handbook of Emotion Regulation, 1(1), 87-109.

Ochsner, K. N., Ray, R. D., Cooper, J. C., Robertson, E. R., Chopra, S., Gabrieli, J. D., \& Gross, J. J. (2004). For better or for worse: Neural systems supporting the cognitive down- and up-regulation of negative emotion. NeuroImage, 23(2), 483-499.

Payer, D. E., Baicy, K., Lieberman, M. D., \& London, E. D. (2012). Overlapping neural substrates between intentional and incidental down-regulation of negative emotions. Emotion, 12(2), 229-235.

Payne, J. D., Jackson, E. D., Hoscheidt, S., Ryan, L., Jacobs, W. J., \& Nadel, L. (2007). Stress administered prior to encoding impairs neutral but enhances emotional long-term episodic memories. Learning and Memory, 14(12), 861-868.

Phan, K. L., Taylor, S. F., Welsh, R. C., \& Ho, S.-H. (2004). Neural correlates of individual ratings of emotional salience: A trialrelated fMRI study. NeuroImage, 21, 768-780.

Phan, K. L., Wager, T., Taylor, S. F., \& Liberzon, I. (2002). Functional neuroanatomy of emotion: A meta-analysis of emotion activation studies in PET and fMRI. NeuroImage, 16(2), 331-348.

Porto, P. R., Oliveira, L., Mari, J., Volchan, E., Figueira, I., \& Ventura, P. (2009). Does cognitive behavioral therapy change the brain? A systematic review of neuroimaging in anxiety disorders. Journal of Neuropsychiatry and Clinical Neuroscience, 21(2), 114-125.

Posner, M. I., \& Petersen, S. E. (1990). The attention system of the human brain. Annual Review of Neuroscience, 13, 25-42.

Putman, P., Hermans, E. J., \& van Honk, J. (2010). Cortisol administration acutely reduces threat-selective spatial attention in healthy young men. Physiological Behavior, 99, 294-300.

Quirk, G. J., \& Gehlert, D. R. (2003). Inhibition of the amygdala: Key to pathological states? Annals of the New York Academy of Sciences, 985, 263-272.

Salehi, B., Cordero, M. I., \& Sandi, C. (2010). Learning under stress: The inverted-U-shape function revisited. Learning and Memory, 17(10), $522-530$.

Sarrieau, A., Dussaillant, M., Agid, F., Philibert, D., Agid, Y., \& Rostene, W. (1986). Autoradiographic localization of glucocorticosteroid and progesterone binding sites in the human post-mortem brain. Journal of Steroid Biochemistry, 25(5B), 717-721.

Schilling, T. M., Kölscha, M., Larra, M. F., Zecha, C. M., Blumenthal, T. D., Frings, C., \& Schächinger, H. (2013). For whom the bell (curve) tolls: Cortisol rapidly affects memory retrieval by an inverted Ushaped dose-response relationship. Psychoneuroendocrinology, 38(9), 1565-1572.

Schneider, F., Grodd, W., Weiss, U., Klose, U., Mayer, K. R., Nagele, T., \& Gur, R. C. (1997). Functional MRI reveals left amygdala activation during emotion. Psychiatry Research, 76(2/3), 75-82.

Schneider, F., Gur, R. C., Gur, R. E., \& Muenz, L. R. (1994). Standardized mood induction with happy and sad facial expressions. Psychiatry Research, 51(1), 19-31.

Shang, J., Fu, Y., Ren, Z., Zhang, T., Du, M., Gong, Q.,...Zhang, W. (2014). The common traits of the ACC and PFC in anxiety disorders in the DSM-5: Meta-analysis of voxel-based morphometry studies. PLOS ONE, 9(3), e93432.

Shulman, G. L., Pope, D. L., Astafiev, S. V., McAvoy, M. P., Snyder, A. Z., \& Corbetta, M. (2010). Right hemisphere dominance during spatial selective attention and target detection occurs outside the dorsal frontoparietal network. Journal of Neuroscience, 30(10), 3640-3651.

Silvers, J. A., Weber, J., Wager, T. D., \& Ochsner, K. N. (2015). Bad and worse: Neural systems underlying reappraisal of high- and lowintensity negative emotions. Social, Cognitive, \& Affective Neuroscience, 10(2), 172-179.

Sripada, R. K., Marx, C. E., King, A. P., Rampton, J. C., Ho, S. S., \& Liberzon, I. (2013). Allopregnanolone elevations following pregnenolone administration are associated with enhanced activation of emotion regulation neurocircuits. Biological Psychiatry, 73(11), 1045-53.

Sudheimer, K. D., Abelson, J. L., Taylor, S. F., Martis, B., Welsh, R. C., Warner, C.....Liberzon, I. (2013). Exogenous glucocorticoids decrease subgenual cingulate activity evoked by sadness. Neuropsychopharmacology, 38, 826-845.

Taylor, C. T., Aupperle, R. L., Flagan, T., Simmons, A. N., Amir, N., Stein, M. B., \& Paulus, M. P. (2014). Neural correlates of a computerized attention modification program in anxious subjects. Social, Cognitive, \& Affective Neuroscience, 9(9), 1379-1387.

Taylor, S. F., Liberzon, I., Fig, L. M., Decker, L. R., Minoshima, S., \& Koeppe, R. A. (1998). The effect of emotional content on visual recognition memory: A PET activation study. NeuroImage, 8(2), 188-197.

Taylor, S. F., Phan, K. L., Decker, L. R., \& Liberzon, I. (2003). Subjective rating of emotionally salient stimuli modulates neural activity. NeuroImage, 18, 650-659.

Tzourio-Mazoyer, N., Landeau, B., Papathanassiou, D., Crivello, F., Etard, O., \& Delcroix, N. (2002). Automated anatomical labeling of activations in SPM using a macroscopic anatomical parcellation of the MNI MRI single-subject brain. NeuroImage, 15, 273-289.

Van Peer, J. M., Roelofs, K., Rotteveel, M., van Dijk, J. G., Spinhoven, P., \& Ridderinkhof, R. (2007). The effects of cortisol administration on approach-avoidance behavior: An event-related potential study. Biological Psychology, 76, 135-146.

Veer, I. M., Oei, N. Y. L., Spinhoven, P., van Buchem, M. A., Elzinga, B. M., \& Rombouts, S. A. R. B. (2012). Endogenous cortisol is associated with functional connectivity between the amygdala and medial prefrontal cortex. Psychoneuroendocrinology, 37(7), 1039-1047.

Watzka, M., Beyenburg, S., Blumcke, I., Elger, C. E., Bidlingmaier, F., \& Stoffel-Wagner, B. (2000). Expression of mineralocorticoid and glucocorticoid receptor mRNA in the human hippocampus. Neuroscience Letters, 290(2), 121-124.

Wirth, M. M., Scherer, S. M., Hoks, R. M., \& Abercrombie, H. C. (2011). The effect of cortisol on emotional responses depends on order of cortisol and placebo administration in a within-subject design. Psychoneuroendocrinology, 36(7), 945-954.

Yehuda, R., Bierer, L. M., Pratchett, L. C., Lehrner, A., Koch, E. C., Van Manen, J. A.,....Hildebrandt, T. (2015) Cortisol augmentation of a psychological treatment for warfighters with posttraumatic stress 
disorder: Randomized trial showing improved treatment retention and outcome. Psychoneuroendocrinology, 51, 589-597.

Yehuda, R., Giller, E. L., Southwick, S. M., Lowy, M. T., \& Mason,

J. W. (1991). Hypothalamic-pituitary-adrenal dysfunction in posttraumatic stress disorder. Biological Psychiatry, 30(10), 1031-1048.

Young, E., \& Korszun, A. (2010). Sex, trauma, stress hormones and depression. Molecular Psychiatry, 15(1), 23-28. 